\title{
Designing tensegrity modules for pedestrian bridges
}

\author{
Landolf Rhode-Barbarigos ${ }^{1, *}$, Nizar Bel Hadj Ali ${ }^{1}$, René Motro ${ }^{2}$ and Ian F.C. Smith ${ }^{1}$ \\ ${ }^{1}$ Applied Computing and Mechanics Laboratory, Structural Engineering Institute, Station 18, Ecole \\ Polytechnique Fédérale de Lausanne (EPFL), Lausanne CH-1015, Switzerland. \\ ${ }^{2}$ Laboratoire de Mécanique et Génie Civil (LMGC), Université de Montpellier II, Montpellier Cedex, France. \\ * Corresponding author. Tel: +41 2169363 72, Fax: +41 2169347 48,
}

Email: landolf-giosef.rhode-barbarigos@epfl.ch

\begin{abstract}
Tensegrity systems are spatial structures composed of tensile and compression components in a selfequilibrated state of prestress. The tensegrity concept has already been studied by researchers in various fields over the past decades. A family of tensegrity modules that can offer promising solutions for civil engineering applications such as tensegrity domes, towers and bridges is analyzed. Research into tensegrity systems has resulted in reliable techniques for form finding and structural analysis. However, the tensegrity concept is not yet part of mainstream structural design. This paper presents a design study of a tensegrity-based pedestrian bridge. Structural performance of the bridge using three tensegrity modules is evaluated through parametric studies. Design requirements for pedestrian bridges and results of parametric studies are used to define a design procedure that optimizes section sizes for this type of structure. A structural efficiency indicator is proposed and used to compare proposals for feasible bridge configurations. Design results illustrate that the hollow-rope tensegrity bridge can efficiently meet typical design criteria.
\end{abstract}

\section{Keywords}

Tensegrity system, Pedestrian bridge, Structural design, Structural efficiency.

\section{Introduction}

Tensegrity systems are a special class of spatial reticulated structures that are composed of struts and tendons. Tensioned and compressed components are assembled in a self-equilibrated system providing stability and stiffness to the structure. The word tensegrity comes from the contraction of tensile and integrity. It was proposed by Richard Buckminster Fuller in 1962. He described tensegrity systems as islands of compression in an ocean of tension. During the same period, Kenneth Snelson and David George Emmerich patented similar systems. A recent and widely accepted definition was proposed in 2003 [1]: "A tensegrity is a system in stable self-equilibrated state comprising a discontinuous set of compressed components inside a continuum of tensioned components". This definition includes systems where compressed elements are interconnected as tensegrity structures [2]. Skelton et al. [3] proposed the term "class $k$ " to distinguish the different types of structures included in this broader definition. A "class $k$ " tensegrity structure is then defined as a stable tensegrity with a maximum of $k$ interconnected compressive members. 
Over the last decades, the tensegrity concept has received significant interest among scientists and engineers throughout disciplines such as architecture, civil engineering, biology, robotics and aerospace [4]. The tensegrity concept offers a high level of geometrical and structural efficiency and results in modular and lightweight structures. However, few examples of tensegrity structures have been used for civil engineering purposes. Paronesso and Passera proposed a tensegrity platform for the 2002 Swiss National Exhibition in Yverdon [5]. They also designed a tensegrity roof for the velodrome in Aigle (Switzerland) [6]. Designed by Schlaich, Bergermann and Partners, the Rostock tower (Germany) built in 2003 is probably the highest tensegrity tower $(62.3 \mathrm{~m})$. The tower is composed of a continuous assembly of six "simplex" modules [7]. Describing the conceptual and structural design of the tower in Rostock, Schlaich [8] concluded that despite their inherent flexibility the potential of tensegrities for tower and roof structures is substantial. Moreover, large tensegrity grids can be constructed by assembling elementary self-stressed modules [9]. Studies on double layer tensegrity grids were initiated by Motro and Hanaor [10]. Hanaor [11] presented design aspects of double layer tensegrity grids. Quirant et al [12] studied the different stages of tensegrity grid design. A design procedure was developed and applied to a double layer tensegrity grid covering a surface of 81 $\mathrm{m}^{2}$.

Research into tensegrity structures revealed that only small amounts of energy are needed to change the shape of such systems. Tensegrities are thus regarded as an attractive solution for controllable and deployable systems [13]. Tibert and Pellegrino [14] compared the stiffness of a deployable tensegrity mast with a conventional mast. They identified lack of stiffness during deployment and weak deployed bending stiffness as obstacles to practical applications. Fest et al. [15] experimentally explored potential of active tensegrities on a five-module tensegrity structure. A quasi-static control strategy based on stochastic search is first proposed to satisfy serviceability criterion $[16,17]$. The control strategy is then extended to take into account additional robustness objectives [18, 19]. Smaili and Motro [20] presented a design study of curved tensegrity systems and investigated the deployment process requirements of such structures. $\mathrm{Vu}$ et al [21] investigated the basic design concept of deployable tension-strut structures. These tensegrity-like structures are compared with conventional double layer truss systems through parametric studies. Vu et al [21] concluded that deployable tensionstrut systems are structurally more efficient than double layered spatial trusses for span up to $48 \mathrm{~m}$. Motro et al [22] proposed a new family of tensegrity cells called "tensegrity rings" that can be assembled in a "hollow rope" and provided a general method for creating these foldable tensegrity cells starting from any n-sided prism. The concept of "hollow rope" shows promise for architecture and civil engineering applications such as pedestrian bridges. The increasing amount of research into tensegrity systems has resulted in reliable techniques for form finding and structural analysis. However, there has been relatively little research on design strategies for structural purposes. Thus, there is a need to develop systematic methods for tensegrity design.

This paper presents a tensegrity "hollow rope" pedestrian bridge designed according to Swiss civilengineering practice. The tensegrity pedestrian bridge is designed by assembling elementary selfstressed modules. Three bridge configurations designed with different elementary modules are studied having the same span, internal space and load cases. A parametric study is performed to identify parameters that significantly affect the behavior of the structure. A comparison of different configurations and a description of the feasible configuration are included. Static analysis is conducted with a dynamic-relaxation algorithm. Finally, an extension of the design method is proposed for tensegrity bridges.

\section{Tensegrity pedestrian bridge}

The proposed structure is a pedestrian bridge to be built in the French speaking part of Switzerland. The bridge spans $20 \mathrm{~m}$ over a river. A distance of $3 \mathrm{~m}$ from the ground is considered. The structural system of the bridge is composed of four identical tensegrity modules (Figure 1). Symmetry about midspan is obtained by mirroring two modules. 


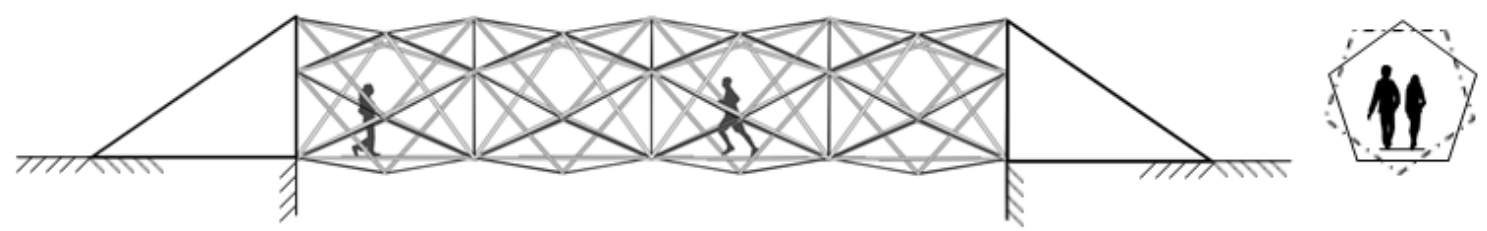

Figure 1: The tensegrity pedestrian bridge

The structure of the bridge is designed with a minimum internal space for walking. The walking area is a rectangular internal free space with a height of $2.5 \mathrm{~m}$ and a width of $1.3 \mathrm{~m}$. These dimensions are chosen to have enough room for two persons crossing the bridge simultaneously. Pedestrians cross the bridge walking on a steel deck supported on the bottom nodes at both ends of each module.

The proposed pedestrian bridge is designed as a tensegrity structure. Therefore, two different stages must be considered when designing this structure: a pre-stress phase and a service phase. During the first phase, self-stresses are applied to the tensegrity structure in order to avoid slack cables and excessive deflections due to dead load. During the service phase the structure is considered to be fully operational. The boundary conditions of the structure are identical on both stages. All nodes at both extremities are blocked in all directions (Figure 2).

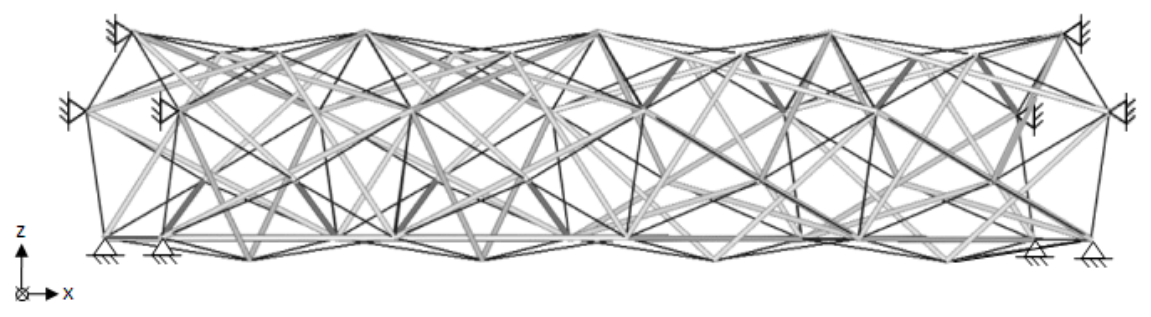

Figure 2: Boundary conditions of the tensegrity bridge

\section{Tensegrity modules}

Designing structurally efficient tensegrity systems for civil engineering application is a challenging task. A wide variety of elementary tensegrity modules can be used to develop structural systems. Modules can be assembled in linear, plane filling and space filling forms. The tensegrity bridge that is studied in this paper is composed of four tensegrity modules each having a $5 \mathrm{~m}$ length. A preliminary study involving various module topologies was conducted. Three module configurations were retained for the tensegrity pedestrian bridge based on superior structural performance. Retained modules are presented and compared in this paper. All three modules were designed and compared considering the bridge geometry requirements for length and minimum pedestrian space.

Pugh [23] proposed elementary tensegrity modules of polygonal form which have important open spaces at their centers. In these modules, each strut end is jointed to another to create circuits of struts within outer cable circuits. Previous work [22] has classified them as "ring modules" because of their hollow tube shape. A generic construction method for ring modules based on the geometry of straight prisms was proposed. This method provides regularity for elementary ring module topology and is illustrated in Figure 3. The topology can be understood through studying the position of the struts: first 
the diagonal struts (Figure 3a) and then the intermediate struts (Figure 3b) according to the vertices of a straight prism. Intermediate struts connect a chosen middle node to two base nodes, one each at the top and bottom faces of the prism. In this study, middle nodes are chosen on the circumscribing circle at mid-length of the module. There are two kinds of cables: layer cables connecting the base nodes of the straight prism (Figure 3c) and x-cables connecting the middle nodes with the base nodes (Figure $3 d)$.

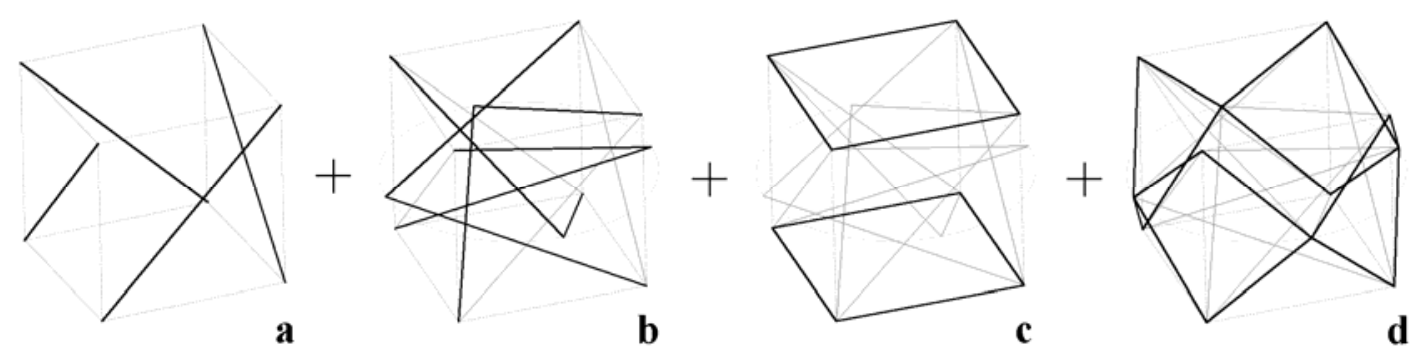

Figure 3: Understanding the topology of the square tensegrity ring module: a) diagonal struts, b) nondiagonal struts, c) layer cables, d) x-cables

The tensegrity ring modules that are studied in this paper are thus called square, pentagon and hexagon modules. Modules are named after the polygon defined by the cables on a module face (y-z plane). Figure 4 shows the cable polygons inside their rings and the corresponding modules. Topologies of the following three ring modules are defined using the construction method proposed in [22].

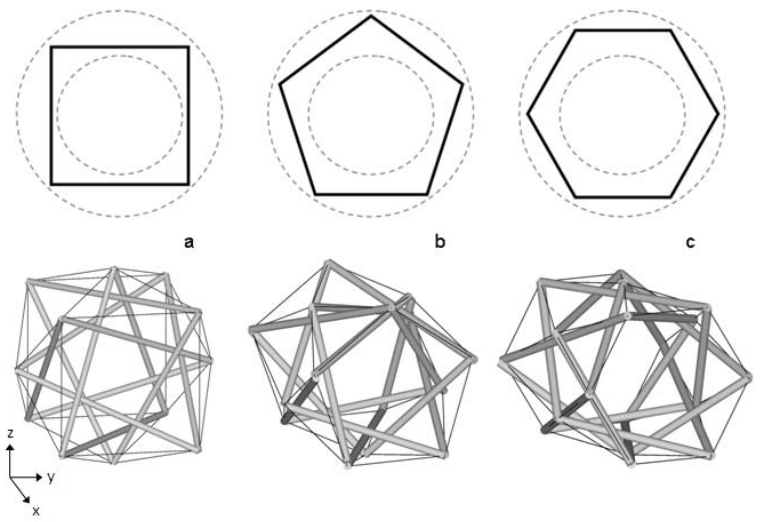

Figure 4: Cable polygons inside the ring: a) square b) pentagon and c) hexagon

The square is a semi-continuous module composed of four triangle strut circuits (Figure 4a). The 12 struts in this module are connected to 24 cables by 12 nodes. The struts have lengths of $6.20 \mathrm{~m}$ and $6.60 \mathrm{~m}$. This module can be enclosed in a ring with an internal radius of $1.15 \mathrm{~m}$ and an external radius of $3.10 \mathrm{~m}$. The pentagon module is a continuous module with a single strut circuit (Figure 4b). The circuit of 15 struts connects to 30 cables through 15 nodes. Its topology includes three pentagons of cables in three different layers. The pentagon struts have lengths of $4.30 \mathrm{~m}$ and $5.60 \mathrm{~m}$. The assembled ring shape for this module has an internal radius of $1.26 \mathrm{~m}$ and an external radius of $2.15 \mathrm{~m}$. Finally, the hexagon is a continuous ring module with a single strut circuit of 18 elements (Figure 4c). Three 
hexagons of cables are created on different planes. Thirty-six cables connect to the 18 struts of the hexagon module in 18 nodes. The struts are $3.80 \mathrm{~m}$ and $5.40 \mathrm{~m}$ long. This module has an internal radius of $1.40 \mathrm{~m}$ and an external radius of $1.99 \mathrm{~m}$.

A main step in the characterization of a tensegrity system is the computation of the number of infinitesimal mechanisms and the number of independent states of self-stress for a pre-stressed configuration. Self-stress states are important because not only they stiffen the structure but they can also stabilize infinitesimal mechanisms. Static and kinematic properties of tensegrity structures can be determined through Singular Value Decomposition (SVD) of the equilibrium matrix [24, 25]. Table 1 shows the results for infinitesimal mechanisms and independent states of self-stress for the ring modules considered in this study. Pre-stressed ring module configurations are studied considering six kinematical constraints so that rigid body displacements are avoided. Continuous ring modules (pentagon and hexagon) have no infinitesimal mechanisms and six independent states of self-stress. The square module has one infinitesimal mechanism with a base of seven elementary self-stress states.

Table 1: Computation of the number of infinitesimal mechanisms and the number of independent self-stress states of the three tensegrity modules

\begin{tabular}{lllllll}
\hline Module & Elements, $b$ & Joints, $j$ & $\begin{array}{l}\text { Equilibrium } \\
\text { matrix rank }\end{array}$ & $\begin{array}{l}\text { Kinematical } \\
\text { constraints, } k\end{array}$ & $\begin{array}{l}\text { Infinitesimal } \\
\text { mechanisms, } m\end{array}$ & $\begin{array}{l}\text { Self-stress } \\
\text { states, } s\end{array}$ \\
\hline Square & 36 & 12 & 29 & 6 & 1 & 7 \\
Pentagon & 45 & 15 & 39 & 6 & 0 & 6 \\
Hexagon & 54 & 18 & 48 & 6 & 0 & 6 \\
\hline
\end{tabular}

\section{Parametric analysis}

I. Analysis method

Although the concept of tensegrity has activated interest in various fields, designing tensegrity systems for civil-engineering applications is not yet part of mainstream structural engineering. Additionally, available design codes and guidelines do not accommodate these structural systems.

Tensegrity structures behave nonlinearly in response to external loading. Since tensegrities are selfstressed and flexible, displacements can be large even for small deformations of critical elements. Therefore, the behavior of this kind of structure is complex and this is further complicated by a significant number of design parameters that have to be taken into consideration. Designing a tensegrity structure could require iterative analyses for successive modifications, such as changes to material properties and element self-stress. A complete analysis of a tensegrity system comprises three steps: form finding, implementation of self-stress and finally, the study of behavior under external loads.

Form finding of a tensegrity system consists of determining a stable self-stressed state. Research into tensegrity form finding resulted in methods such as nonlinear programming techniques [26], dynamic 
relaxation [27], force density [28], and stochastic search [29]. A review of these and other methods can be found in $[30,31]$. In general, these methods require a valid topology to start the process of finding a stable configuration. When a stable configuration is identified, the self-stress level in the tensegrity system can be increased by choosing either cable lengths smaller or strut lengths longer than the geometrical distance between nodes according to the topology of the structure [32].

Another important aspect of the behavior of tensegrity structures is geometric nonlinearity. Nonlinear modeling and iterative computational schemes are needed to design tensegrities. In this context, two approaches have been developed and applied in practice. The first one is a standard nonlinear structural analysis where the static equilibrium equation is solved incrementally using modified Newton-Raphson iterative procedure [32]. The second is dynamic relaxation which was first introduced by Day [33] and has been reliably applied to many tensile structures [27], tensegrities and other nonlinear problems. The dynamic relaxation method is an iterative procedure used to find the static equilibrium state of a structure. A fictitious dynamic model is used to trace the motion of a structure from the moment of loading to the moment of static equilibrium attained due to damping [27].

In this study, static analyses of tensegrity bridge configurations are performed using dynamic relaxation with kinetic damping. The maximum kinetic energy peak, determined through the calculation of consecutive nodal velocity vectors at each time increment, is chosen as new reference state. All nodal velocities are then reset to zero and the calculations are continued until the unbalanced forces converge to zero. The dynamic relaxation method does not require an assembled stiffness matrix. Moreover, its equations are always expressed in terms of current coordinates of the structure. Therefore, it is particularly suitable for structures having geometrical nonlinearities.

\section{Parametric study}

Parametric studies help only to estimate the influence of different design parameters on the structural behavior of the tensegrity bridge and do not necessarily include feasible configurations. The study is carried out for the three elementary configurations of ring modules. These configurations have identical element cross-sectional areas and self-stress level. Additionally, they sustain identical service loads. Design loading includes the dead load of elements except joints, pedestrian loads and a horizontal load. Each configuration is composed of four identical modules and has the same boundary conditions.
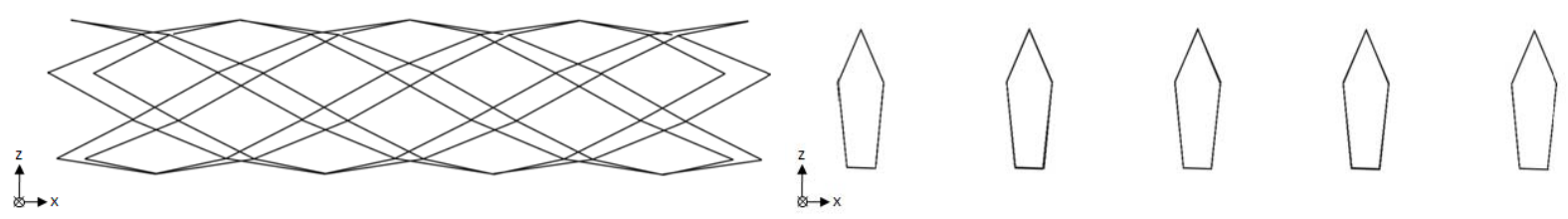

Figure 5: Illustration of the x-cables (left) and layer cables (right)

Five parameters are examined: the cross-sectional area of $\mathrm{x}$-cables, the cross-sectional area of layer cables, the cross-sectional area of struts, the rigidity ratio strut-cable and the self-stress. X-cables are cables that envelop the struts, while layer cables are found at the ends of every module (Figure 5). Following standard practice analysis, a range of values for every parameter is studied while other parameters are left unchanged. The analysis thus reveals the influence of each parameter assuming 
mutual independence. Table 2 shows the values for the default configuration of the structure. All configurations have a $5 \%$ self-stress with respect to the tensile resistance of cables. This relatively low self-stress level is deliberately chosen, since a high initial self-stress may not reveal the individual influence of each parameter. The average vertical displacement at midspan is chosen to compare the structural performance of the configurations. In the next sections, results related to each design parameter are presented.

Table 2: Default configuration for the parametric study

\begin{tabular}{ll}
\hline Parameter & Values \\
\hline Layer cable cross-section & $1.5 \mathrm{~cm}^{2}$ \\
X-cable cross-section & $1.5 \mathrm{~cm}^{2}$ \\
Strut cross-section & $6.0 \mathrm{~cm}^{2}$ \\
Cable elastic modulus & $115 \mathrm{GPa}$ \\
Strut elastic modulus & $210 \mathrm{GPa}$ \\
\hline
\end{tabular}

a. Cross-sectional area of $\mathrm{x}$-cables

X-cables create a longitudinal envelop for the bridge around the struts (Figure 5). Values of crosssectional area for $\mathrm{x}$-cables are presented in Table 3.

Table 3: Parametric values of cables

\begin{tabular}{cccccc}
\hline Parameter & \multicolumn{5}{c}{ Cross-sectional area } \\
\hline $\mathrm{A}\left[\mathrm{cm}^{2}\right]$ & 0.28 & 0.75 & 1.50 & 3.00 & 6.00 \\
\hline
\end{tabular}




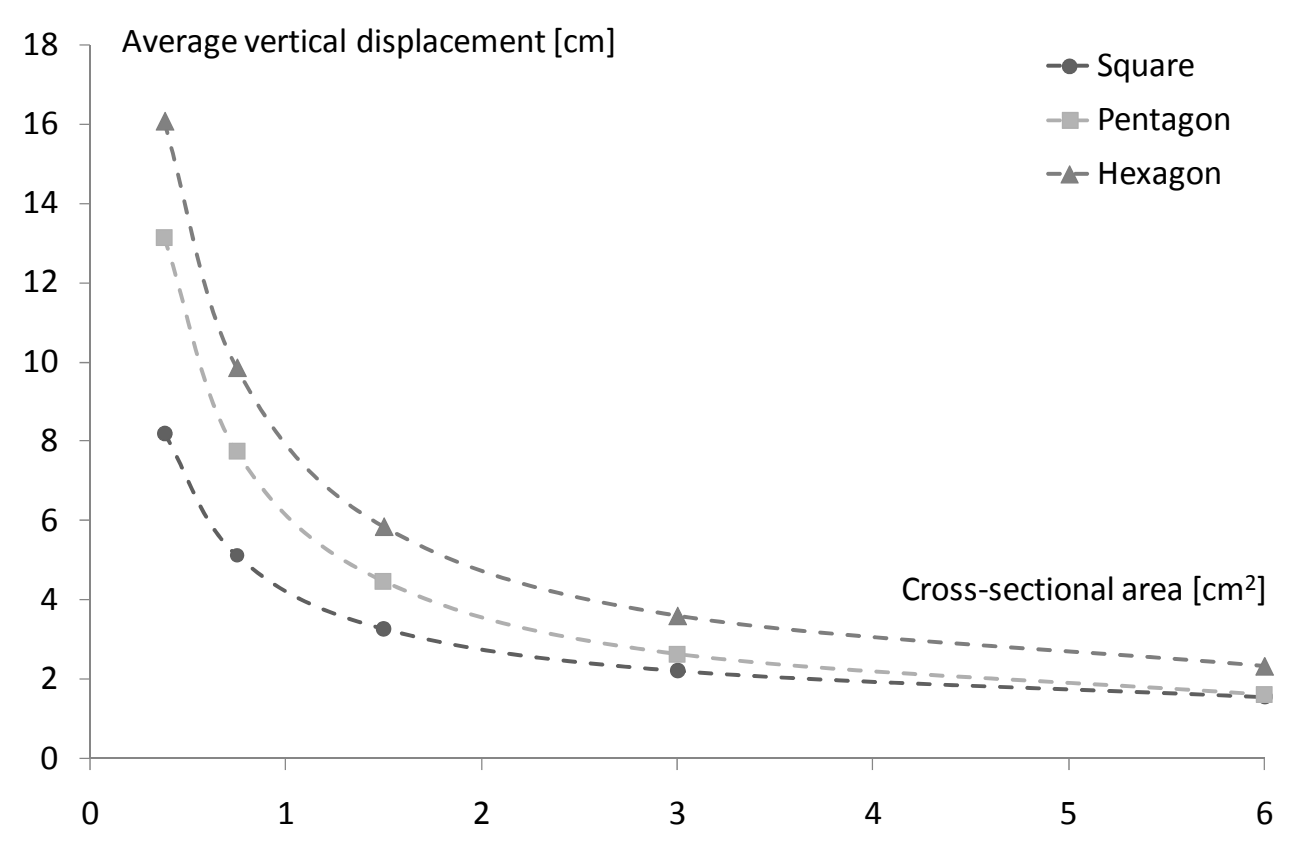

Figure 6: Parametric analysis on the cross-sectional area of $x$-cables

The evolution of the average vertical displacement at mid-span with respect to the cross-sectional area of x-cables is illustrated in Figure 6 for all three configurations. Results illustrated in this figure show a common trend for the three modules: increasing the cross-sectional area of x-cables decreases the average vertical displacement at mid-span. An increment of the cross-sectional area of the x-cables results in an increased stiffness of the structure without significantly increasing dead loads. An observation emerging from the parametric analysis of the cross-sectional area of $\mathrm{x}$-cables is that there is a difference in the magnitude of displacements between the three modules. This difference can be attributed to the additional weight that is introduced by the presence of additional elements.

\section{b. Cross-sectional area of layer cables}

Layer cables are positioned on the two polygonal faces of every module. Thus, the tensegrity bridge composed of four modules contains layer cables that form five polygons along its longitudinal axis (Figure 5). The values of cross-sectional area considered for layer cables are identical to x-cable values (Table 2). 


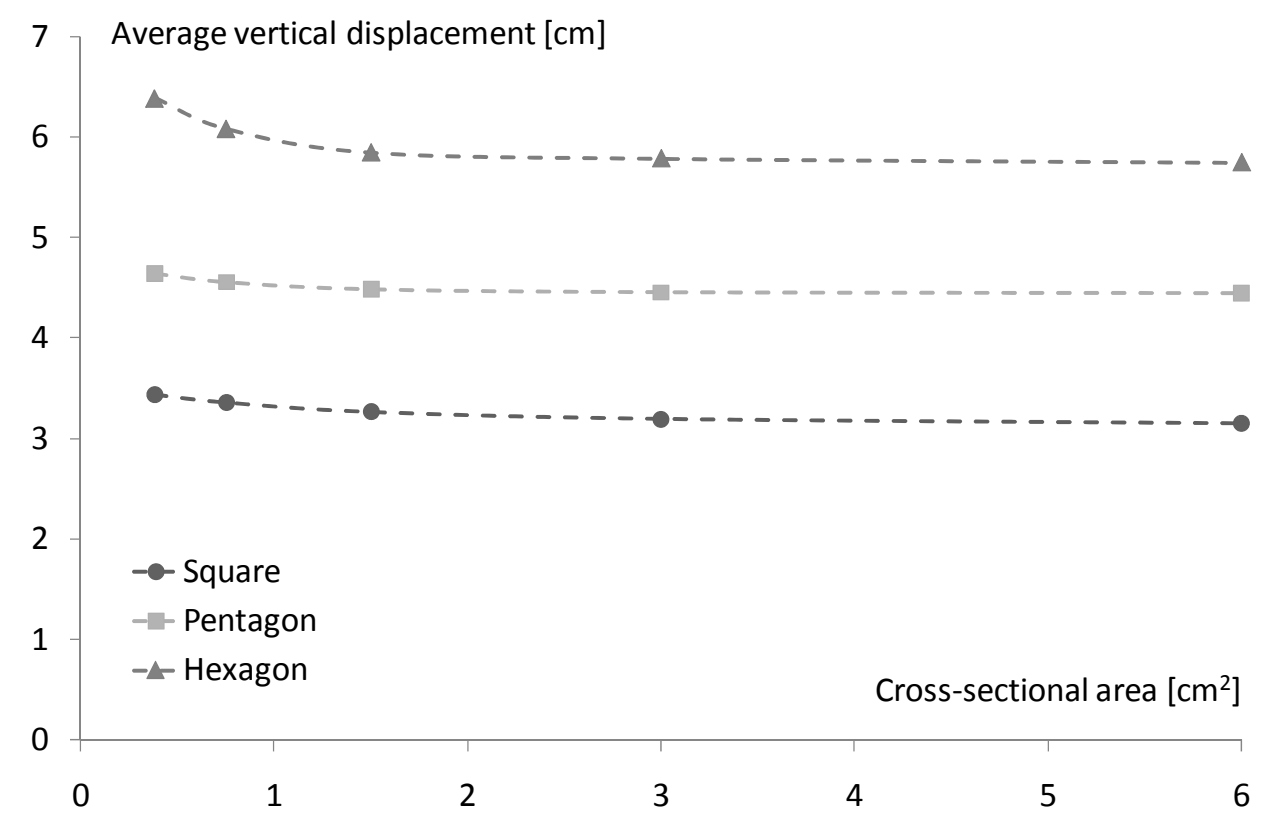

Figure 7: Parametric analysis on the cross-sectional area of layer cables

Average vertical displacement at the mid-span section of the bridge is illustrated with respect to the cross-sectional area of layer cables in Figure 7. Once again there is a difference in the magnitude of displacements between the three modules. For the three configurations of the bridge, the behavior of the structure suggests that increasing the cross-sectional area of layer cables marginally decreases the vertical displacement until reaching a limit. In this case, the reduction on the displacement magnitude due to an increment of the cross-sectional area of layer cables is smaller than the one observed due to $\mathrm{x}$-cables.

\section{c. Cross-sectional area of struts}

Ring modules have a continuous strut circuit and therefore have a higher bending stiffness compared to modules that do not have a continuous strut circuit. Struts are placed respecting a rotational regularity around the longitudinal axis of the module. However, there is no symmetry in any y-z plan (Figure 8). The three configurations of the bridge are analyzed using different values for strut crosssectional areas. Five values are considered in this parametric study and are displayed in Table 4.

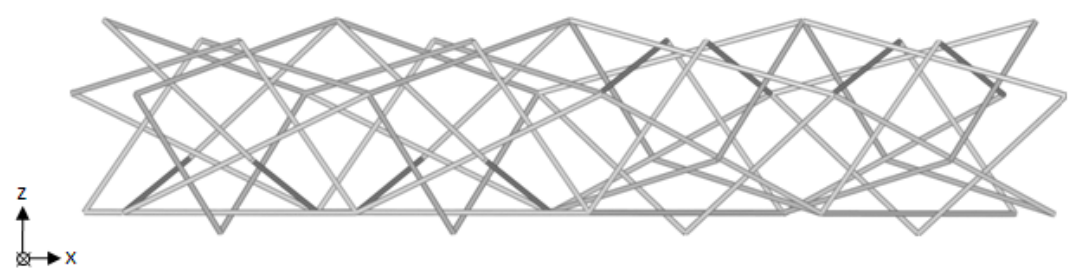

Figure 8: Illustration of struts for the four pentagon module bridge 
Table 4: Parametric values of struts

Parameter Cross-sectional area

\begin{tabular}{llllll}
\hline $\mathrm{A}[\mathrm{cm} 2]$ & 1.50 & 3.00 & 6.00 & 12.00 & 24.00 \\
\hline
\end{tabular}

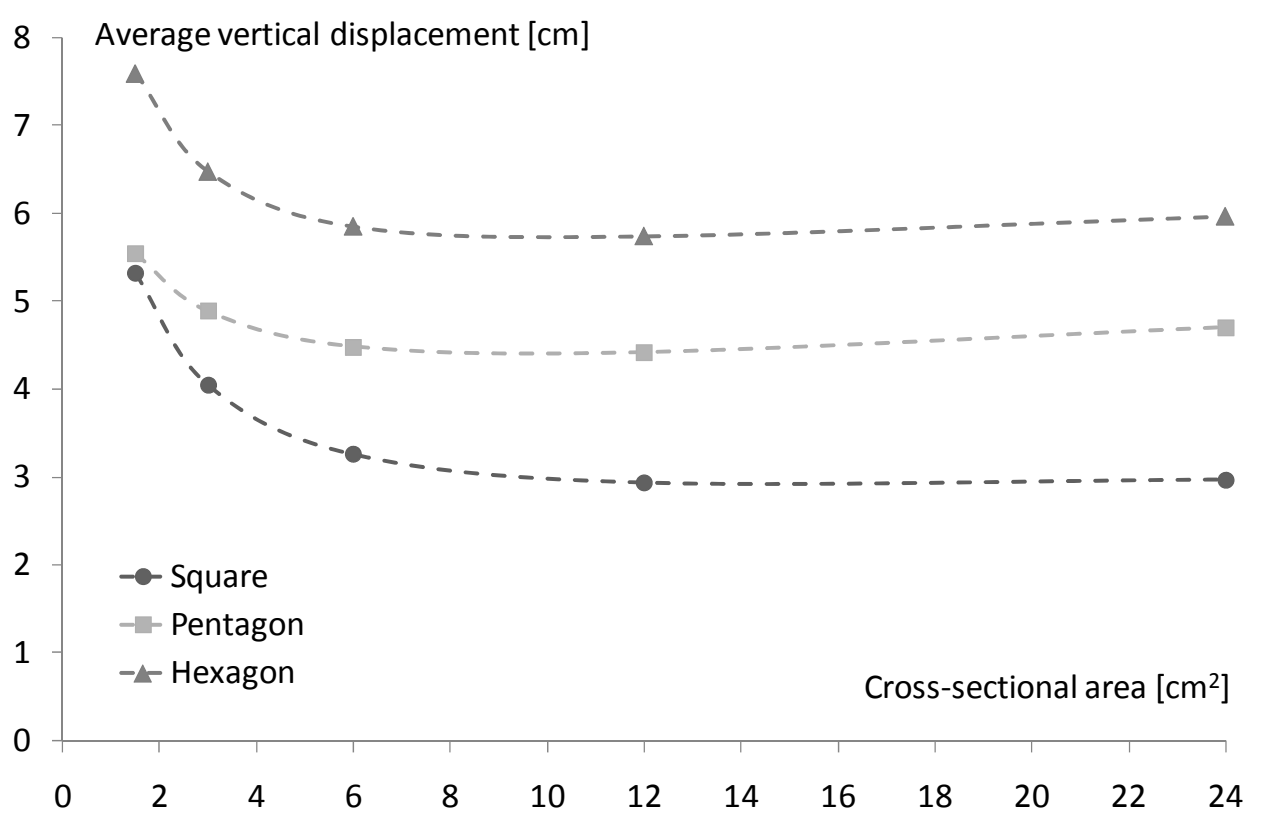

Figure 9: Parametric analysis on the cross-sectional area of struts

Displacement results in relation with the cross-sectional area of struts are shown in Figure 9. Differences in the magnitude of displacements between the three modules are again observed. Increasing the cross-sectional area of the struts decreases the vertical displacement at mid-span until reaching a cross-sectional area of approximately $12 \mathrm{~cm}^{2}$. For all configurations the reduction in displacement becomes insignificant beyond this value. In fact, increasing the cross-section of the struts higher than this value induces a small increment in the vertical displacement at midspan. This suggests that the increase in the stiffness of the structure is counter-balanced by an equivalent increase in dead loads.

\section{d. Rigidity ratio cables - struts}

The structural elements of a tensegrity system experience only axial loading. Tensioned and compressed elements are assembled in a self-equilibrated system providing stability and stiffness to the structure. Cable and strut characteristics were studied separately in previous sections. Since the stiffness of a tensegrity is made by the contribution of both cable and strut stiffness, the influence of 
the rigidity ratio between tensioned and compressed members on the behavior of the tensegrity structure was studied. The rigidity ratio includes the elastic axial rigidity expressed in Eq. (1).

$$
k=\frac{E A}{L}
$$

where $E$ is the modulus of elasticity, $A$ is the cross-sectional area and $L$ is the length of the element. Cross-sectional areas are identical for all three configurations. Consequently, the ratio depends on the modulus of elasticity and the length of the elements. The rigidity ratio is defined in Eq. (2).

$$
\text { Rigidity ratio }=\frac{k_{\text {cables }}}{k_{\text {struts }}}
$$

In order to study the influence of the rigidity ratio on the average vertical displacement, five modulus of elasticity are taken into account for cables while the modulus of elasticity is kept constant for struts. Table 5 shows values considered for cable modulus of elasticity.

Table 5: Parametric values of modulus of elasticity of cables

Parameter Modulus of elasticity

$\begin{array}{llllll}\mathrm{E}[\mathrm{GPa}] & 10 & 50 & 115 & 150 & 210\end{array}$

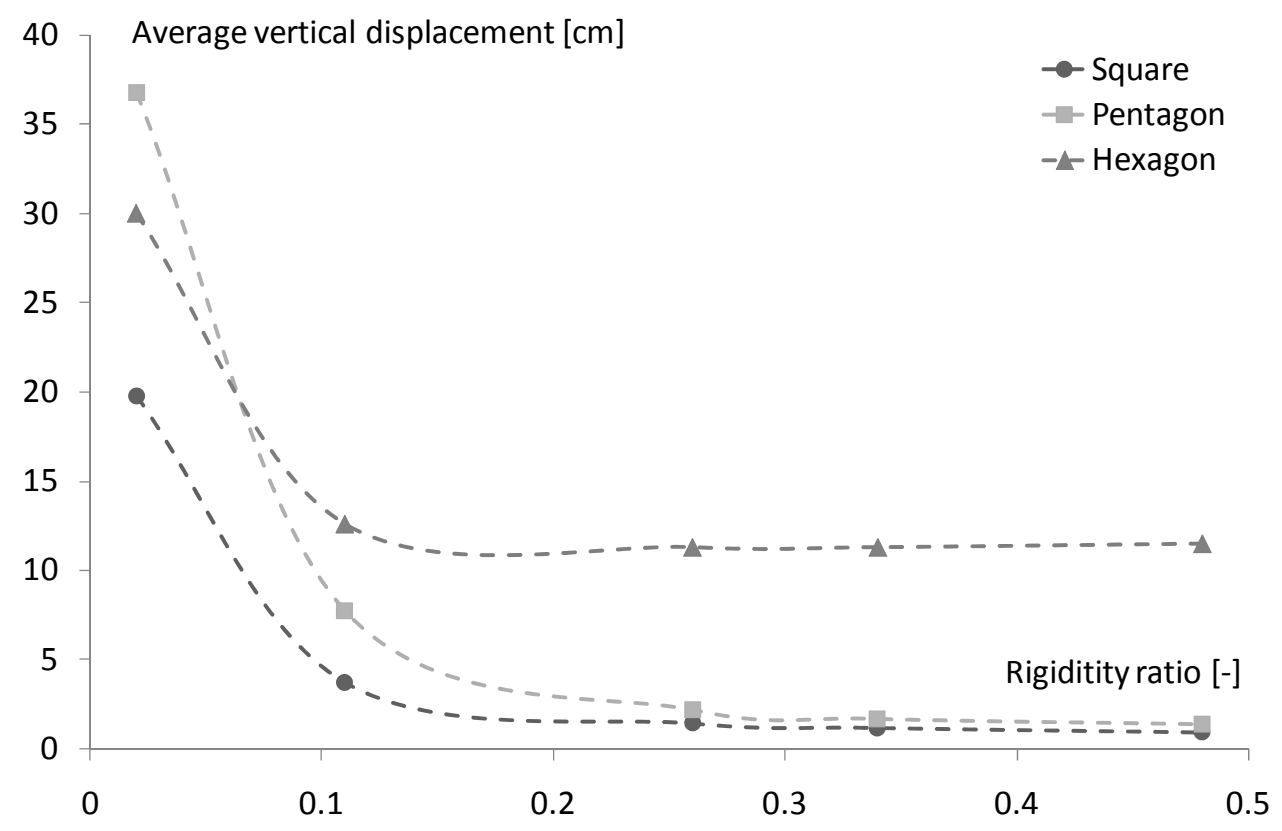

Figure 10: Parametric analysis on the rigidity ratio of steel struts and cables 
For the three configurations of the bridge, the average displacement at midspan is calculated for a range of rigidity ratios (Figure 10). The evolution of midspan displacement with respect to the ratio of rigidity between cables and struts reveals a common tendency for the three modules. In all three configurations increasing the rigidity ratio results in a decrease in the average vertical displacement at midspan. However, the reduction in displacement becomes small beyond a certain value for the rigidity ratio. Consequently, an optimal rigidity ratio between tensile and compressive elements can be identified for each configuration. This optimal value can be used to guide the design of a tensegrity structure. For a given configuration identifying a reference rigidity ratio can lead to a good choice for element cross-sectional areas. For example, if design values for cross-sectional areas of struts and cables lead to a smaller rigidity ratio compared with the reference ratio, this leads to a flexible structure having large deflections. Similarly, a rigidity ratio that is higher than the reference ratio provides extra stiffness without a significant reduction in deflection.

\section{e. Self-stress}

Another important parameter of tensegrity structures is self-stress. The self-stress state is responsible for the stability and the high resistance of tensegrity structures. Self-stress can be created in many ways. In this study, cables are assumed to be pre-tensioned. Consequently, tensile stresses are induced in cables while compression occurs in the struts. Pre-stress is specified in terms of tensile ratio: a ratio between the pre-stressing internal force and the tensile strength of the cable (Table 6). From a global point of view, element self-stresses contribute to the overall rigidity of the structure. However, selfstress acting on structural elements reduces their local capacity.

Table 6: Parametric values of self-stress

\begin{tabular}{cccccc}
\hline Parameter & \multicolumn{5}{c}{ Self-stress (\% of cable tensile strength) } \\
\hline$\alpha[\%]$ & 0 & 25 & 50 & 75 & 100 \\
\hline
\end{tabular}




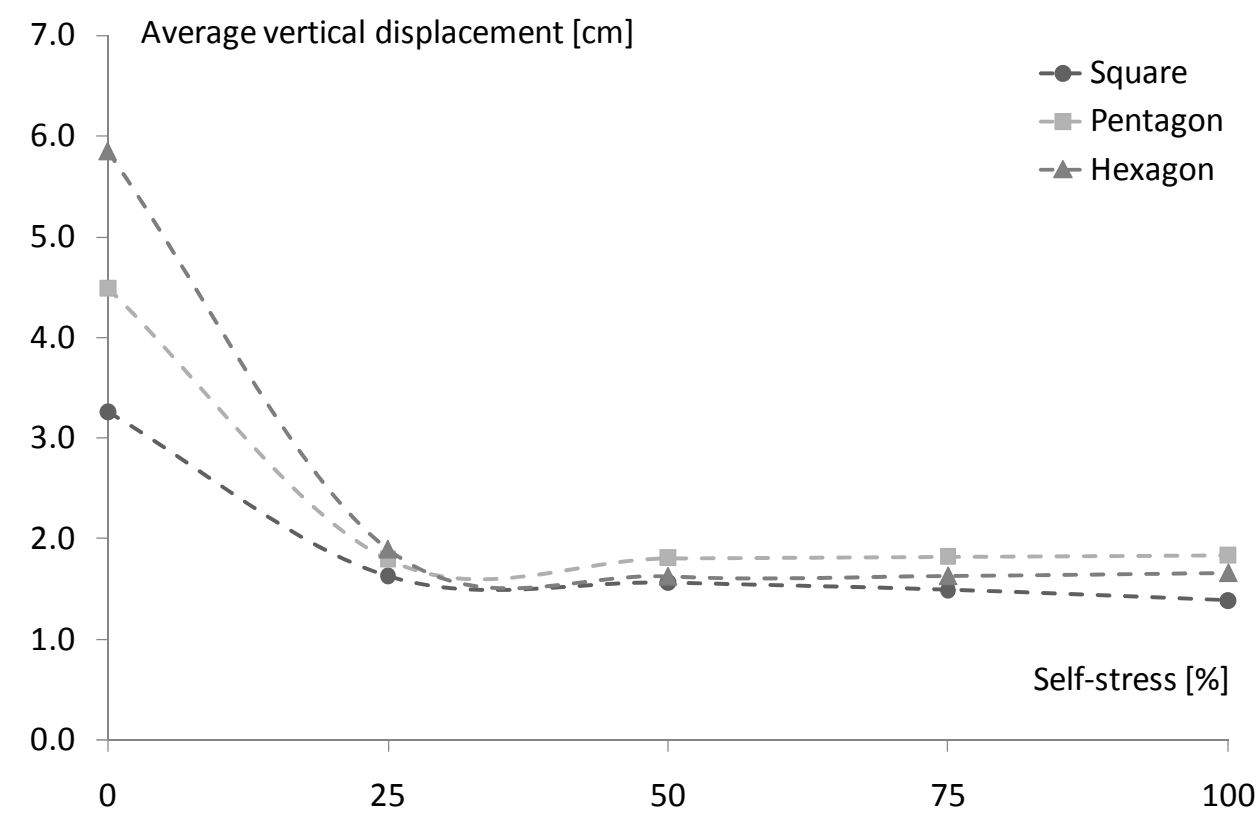

Figure 11: Parametric analysis on the self-stress

Average displacement at the mid-span section of the bridge is illustrated with respect to the self-stress level in relation with the tensile strength in Figure 11. In this case, differences in the magnitude of displacements between the three modules are observed only for values of self-stress smaller than $25 \%$. The evolution of midspan displacement with respect to the level of self-stress reveals also a common tendency: increasing self-stress has a positive influence on the displacement until reaching a plateau. The plateau starts at a self-stress level of approximately $25 \%$ of cable tensile strength for the three modules.

\section{Design procedure}

\section{Design criteria}

The parametric study showed that a number of design parameters influence the behavior of the pedestrian bridge. Designing a tensegrity bridge could require repeated analyses followed by modifications. Material properties, self-stress level and rigidity ratio have to be chosen to ensure safety and serviceability of the structure.

Most modern structural codes, such as the SIA Swiss codes, define safety and serviceability criteria as well as load combinations for footbridge design. Safety criteria ensure overall stability and include verifications of element resistance and stability. Element-resistance checks include tensile strength for tension elements and buckling strength for compression elements (Appendix B). A slenderness limit is also specified for compressive elements in order to avoid local instabilities. The serviceability limitstate requirements for a pedestrian bridge involve limiting vertical displacement at mid-span to satisfy functionality and user comfort as well as appearance criteria. All three criteria are related to the bridge span and should be satisfied simultaneously (Appendix B). Pedestrian bridges should be checked for vibration serviceability if the fundamental frequencies for vertical modes are between 1.6 and $4.5 \mathrm{~Hz}$. 
Analytical studies on these structures have shown that fundamental frequencies are not in this range. Therefore, dynamic aspects are not considered here; this study is limited to static design criteria. Load combination and limit-state checks are given in Appendix B.

\section{Design procedure}

In a study of tensegrity-structure design, Quirant [12] proposed a design procedure that was applied in the case of a double layer tensegrity grid. In a similar approach and based on the conclusions of the parametric study presented in previous sections, a design procedure is proposed and tested for the tensegrity bridge (Figure 12). In this procedure, the design task is guided by the rigidity ratio between tensile and compressive elements. For a fixed geometry with a given choice of constructional materials, member lengths are fixed so that the rigidity ratio depends only on element cross-sectional areas. Once the rigidity ratio is fixed, cross-sectional areas of structural elements are determined iteratively.

The design procedure starts with a pre-defined topology for a tensegrity ring module. Once the topology and nodal positions are known, constitutive materials need to be chosen. The material choice influences the self-weight and the rigidity of the structure. Thus, it is also important for the rigidity ratio between tensile elements and compressive elements. The design rigidity ratio is a reference ratio and it has to be defined for a peculiar topology. This ratio can be estimated from a parametric analysis on the Young modulus of either cables or struts. A rigidity ratio below the design ratio leads to a flexible structure with large deflections. On the other hand, a higher rigidity ratio provides high stiffness with a slight gain in deflections. The next step in the design procedure is to choose a value for the cross-sectional area of struts. Strut area is governed by the slenderness limit of 200 . The reference rigidity ratio permits the calculation of the corresponding cross-sectional area for cables. Thus, the geometry of the structure is defined according to the design rigidity ratio.

Self-stress requires a particular attention during the design due to its dual action as a load for the elements and a rigidity amplifier for the entire structure. A homogeneous self-stress is preferred in this study. When self-stresses are equally distributed on a structure, there is only a minor variation in internal tensile forces when changing the rigidity ratio. First, an analysis under self-weight is conducted in order to verify if both sections and self-stress state need to be modified. If deflection is over the allowable limits then there are two scenarios. High deflection may require the definition of higher sections for the elements. On the other hand, if the difference between calculations and the allowable deflection is small then an increase in self-stress may be enough to satisfy criteria. In cases where deflection is below allowable values, the design can continue to the next stage.

The structure is then analyzed according to serviceability and ultimate limit-state criteria. If one of the criteria is violated, then new cross-sectional areas have to be defined. A sensitivity study should also be conducted in order to evaluate the consequences of slight variations and manufacturing errors [12]. Although, this study may provide additional information on the procedure and the accuracy required, a sensitivity study is not within the scope of this paper. 


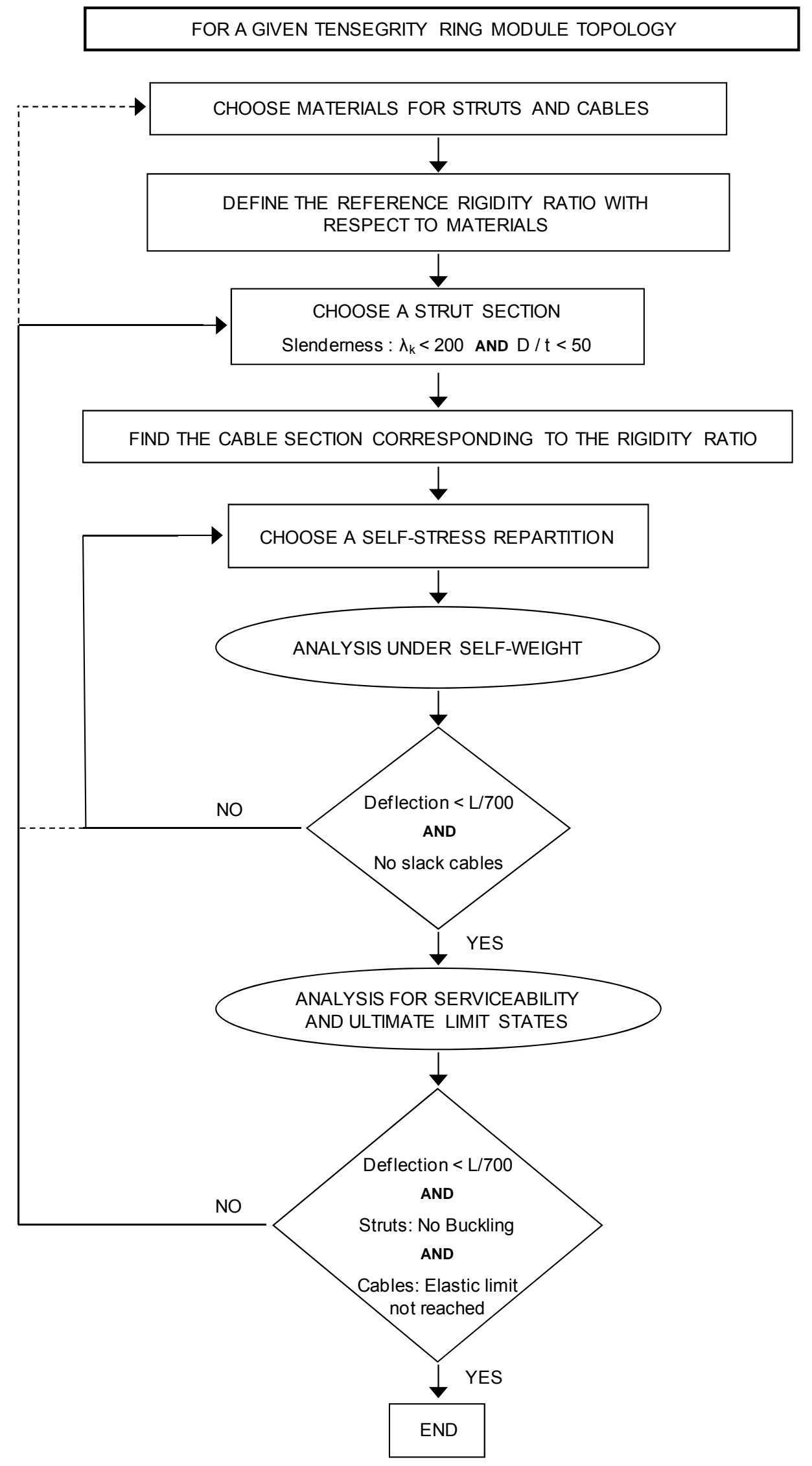

Figure 12: Design procedure for tensegrity ring structures 


\section{Feasible configurations}

The design procedure provides feasible configurations using the three tensegrity ring modules. For the three configurations, steel cables with a Young modulus of $115 \mathrm{GPa}$ are used. Hollow tubes having a Young modulus of $210 \mathrm{GPa}$ are used for compressive components. Required levels of self-stress (10\% with respect to the tensile resistance of cables) for the different configurations are similar. Feasible configurations obtained using the proposed design procedure are presented in Table 7 where $D$ is the strut diameter, $t$ is the strut thickness, $I$ is the strut moment of inertia, $i$ is the strut radius of gyration and $A$ is the cross-sectional area for each element. Buckling strength is the critical design criterion for all three configurations.

Table 7: Feasible configurations of the tensegrity bridge

\begin{tabular}{lllllll}
\hline & $\begin{array}{l}\mathrm{D} \times \mathrm{t} \\
{[\mathrm{mm} \times \mathrm{mm}]}\end{array}$ & $\begin{array}{l}\mathrm{A}_{\text {struts }} \\
{\left[\mathrm{mm}^{2}\right]}\end{array}$ & $\begin{array}{l}\mathrm{I} \\
{\left[\mathrm{mm}^{4}\right]}\end{array}$ & $\begin{array}{l}\mathrm{i} \\
{[\mathrm{mm}]}\end{array}$ & $\begin{array}{l}\mathrm{A}_{\text {x-cables }} \\
{\left[\mathrm{mm}^{2}\right]}\end{array}$ & $\begin{array}{l}\mathrm{A}_{\text {layer cables }} \\
{\left[\mathrm{mm}^{2}\right]}\end{array}$ \\
\hline Square & $114.3 \times 3.6$ & 1250 & $1.920 \times 10^{6}$ & 39.20 & 300 & 100 \\
Pentagon & $101.6 \times 3.6$ & 1110 & $1.330 \times 10^{6}$ & 34.70 & 300 & 100 \\
Hexagon & $95.0 \times 3.2$ & 923 & $0.973 \times 10^{6}$ & 32.50 & 300 & 100 \\
\hline
\end{tabular}

\section{Structural efficiency}

The structural efficiency of tensegrity ring modules is compared in order to identify the best design of the tensegrity pedestrian bridge. Although all three ring modules studied in this paper have some similarities, they also have differences, such as the number of elements and the length of elements. Therefore, in order to have a fair comparison, the bridge configurations are designed using the same procedure and under similar conditions (self-stress, loads, boundary conditions, etc.).

A Structural Efficiency Index (SEI) is used for the comparison of the proposed tensegrity ring modules. This index is inspired from work conducted by Vu et al. [21]. The index is composed of three parts and takes into account design loads, self-weight, span and maximum deflection:

$$
S E I=\alpha \times \beta \times \gamma
$$

where

$$
\alpha=\frac{L+W}{W}, \quad \beta=\frac{S}{D} \text { and } \gamma=\frac{L}{D}
$$

$\mathrm{L}$ is the sum of design loads without their corresponding partial factors, $\mathrm{W}$ the self-weight, $\mathrm{S}$ the span of the bridge and D the maximum deflection at midspan. A high SEI corresponds to a high structural efficiency. For the studied case, design loads are calculated according to the span and the internal 
space of the bridge that are identical for every configuration. Consequently, design loads are also the same.

The first part of SEI reflects the efficiency according to the self-weight of the structure. It includes only the self-weight and loads applied to the structure. These parameters are significant especially for short span structures. In this case, design is usually guided by strength requirements (ULS) rather than serviceability constrains (SLS). The second part of SEI is a common criterion for serviceability including span over deflection. The serviceability limit for this bridge is set to $\mathrm{S} / \mathrm{D}=700$. The last part of the proposed index involves the overall rigidity of the structure through relating "pay load" and deflection. The values for the pentagon module are used to normalize values of SEI (Table 8).

Table 8: Structural Efficiency Index, normalized by values for the pentagon module

\begin{tabular}{|c|c|c|c|c|}
\hline & $\alpha$ & $\beta$ & $\gamma$ & SEI \\
\hline & $\alpha_{\text {pentagon }}$ & $\beta_{\text {pentagon }}$ & $\gamma_{\text {pentagon }}$ & $S E I_{\text {pentagon }}$ \\
\hline Square & 1.00 & 0.45 & 0.45 & 0.20 \\
\hline Pentagon & 1.00 & 1.00 & 1.00 & 1.00 \\
\hline Hexagon & 1.30 & 0.54 & 0.54 & 0.38 \\
\hline
\end{tabular}

Results presented in Table 8 show that the pentagon bridge presents the highest SEI. The pentagon module offers the highest structural efficiency regarding two of the three SEI components. Compared to square and hexagon configurations, the pentagon has a better serviceability performance $(\beta)$ and a higher overall rigidity $(\gamma)$. The square configuration presents a self-weight efficiency $(\alpha)$ that is almost as high as the pentagon, while the hexagon is the most self-weight efficient configuration. However, the serviceability performance and overall rigidity of the hexagon are low. Finally, the square is the less efficient configuration.

\section{Discussion}

I. Differential displacements: torsion

The analysis revealed similar trends on the average vertical displacement at mid-span for the three modules. However, vertical displacements at the mid-span pentagon are not equally distributed. Figure 13 shows the vertical displacements of the nodes on the mid-span polygon for the parametric analysis of the cross-sectional area of $\mathrm{x}$-cables of the pentagon configuration (Figure 5). The torsional effect on the mid-span pentagon is also illustrated. A common trend for all nodes can be identified: increasing the cross-sectional area decreases the average vertical displacement at mid-span. However, the magnitude of the displacements is not the same for the five nodes. Differential displacements at midspan reveal the presence of a torsional effect on the structure. The tensegrity pedestrian bridge is composed of cables and struts. While elements do not experience torsion, the structure of the tensegrity bridge sustains torsion when studied as a whole. 


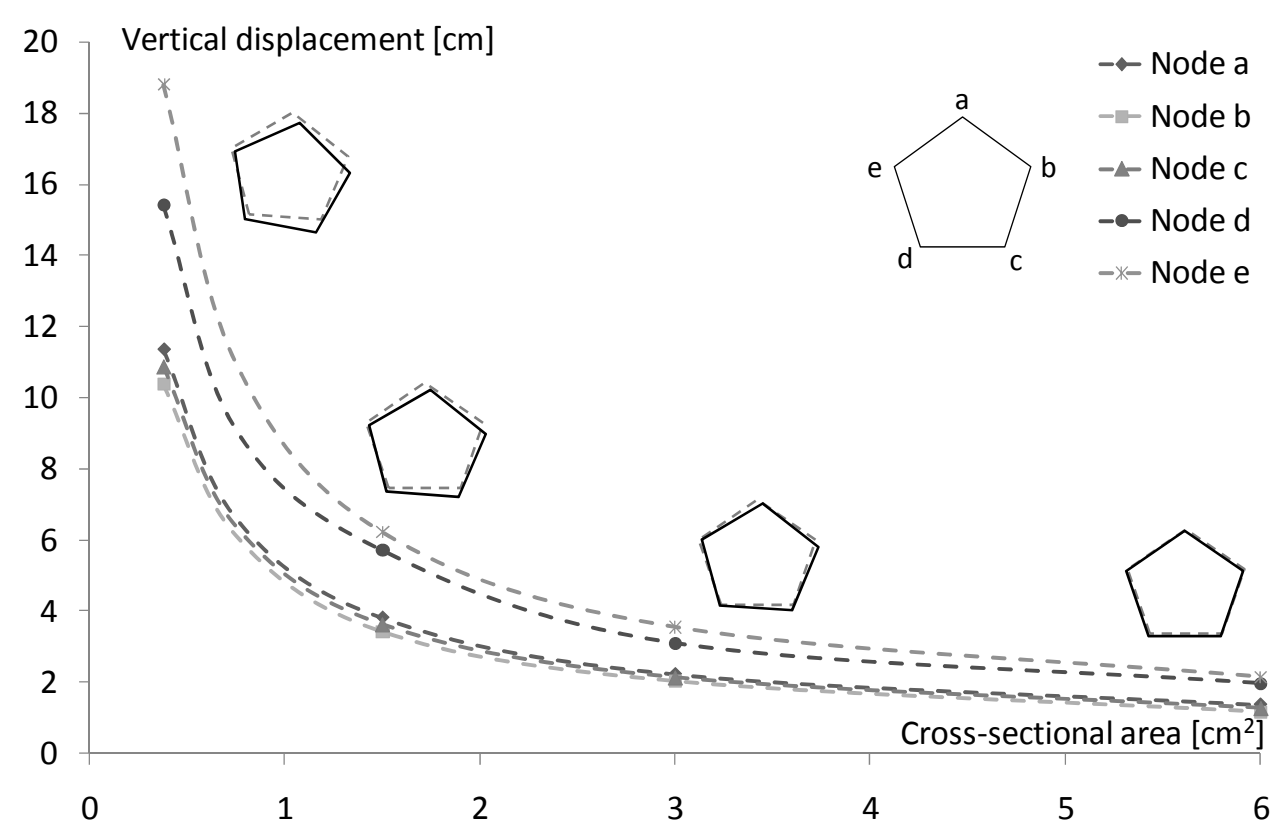

Figure 13: Torsional effect on the mid-span pentagon with respect to the cross-sectional area of $x$-cables

Ultimate limit-state loads applied to the structure are symmetric. Consequently, the torsional effect is only caused by the non-symmetric geometry of the structure. The structure is built according to a method which positions the struts in the shape of a helix. Although there is a symmetry about the longitudinal axis of the bridge ( $\mathrm{x}$ axis) at midspan, there is a lack of symmetry about the y-z plane. Struts rotate around the longitudinal axis of the structure (Figure 14). This rotation make the module behave like a helix. When a load is applied on a helix, the helix has the tendency to twist. This explains the difference of vertical displacements observed on the bridge. The parametric study showed that torsion effects can be avoided with an appropriate sizing of the structural elements. For a given cross-sectional area of x-cables, increasing layer cable and/or strut cross-sectional area can lead to configurations with a lower difference in nodal displacements. However, there are other potential solutions to decrease torsion effects such as non-uniform self-stress or the use of an active control system.

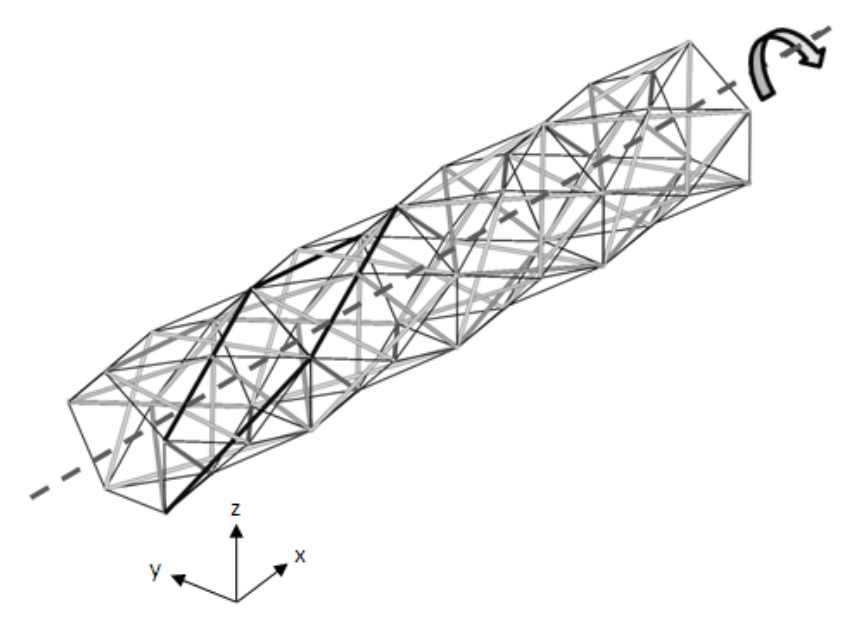

Figure 14: Struts rotating around the longitudinal axis of the bridge 
The effect of torsion can also be a criterion for choosing the best module. Rotation trends increase as the geometry goes from a pentagon structure to a hexagon structure. Thus, the hexagon bridge presents higher differential displacements (higher torsion). This is a supplementary reason to select the pentagon module as the best module for this application.

II. Rigidity ratio design for the pentagonal bridge

The rigidity ratio between tensile elements and compressive elements was identified as an important parameter that can guide the design of a tensegrity structure. Therefore, an additional study with three materials for struts was conducted for the pentagonal configuration. Three modulus of elasticity were considered for steel $\left(\mathrm{E}_{\mathrm{s}}=210 \mathrm{GPa}\right)$, composite (Fiberline with $\mathrm{E}_{\mathrm{F}}=100 \mathrm{GPa}$ ) and a third material with an intermediate value $\left(E_{i}=23 \mathrm{GPa}\right)$. For cables, five elastic modulus values were taken (Table 5).

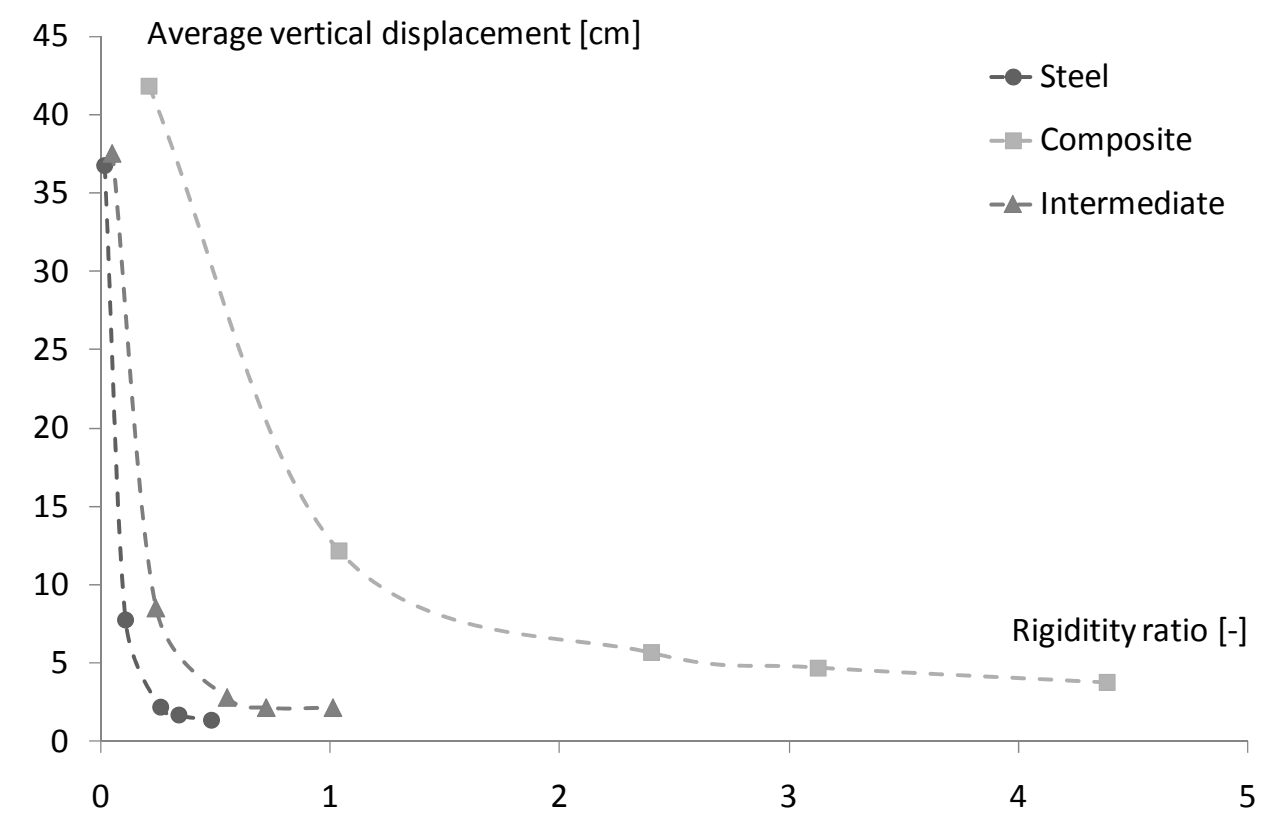

Figure 15: Parametric analysis on the rigidity ratio of struts and cables for the pentagon geometry

It was found that the evolution of average vertical displacement at mid-span with respect to the ratio of rigidity between cables and struts reveals a common trend for the three materials (Figure 15). An increment of the rigidity ratio results in a decrease in the average vertical displacement at mid-span. However, beyond a certain value for the rigidity ratio the reduction in displacement becomes insignificant. Consequently, an optimal rigidity ratio between tensile and compressive elements can be identified not only for each configuration but additionally for every material.

\section{Other criteria for module selection}

The structural analysis of proposed structures revealed that the pentagon is the most suitable module for the bridge application. The pentagon bridge has the overall highest structural efficiency including criteria such as self-weight, serviceability and rigidity. This is due to its topology and to the continuity in its strut circuit. 
The rotation rhythm of struts around the longitudinal axis increases as $n$ increases for any $n$-gone ring module geometry. Thus, the pentagon bridge has the lowest differential displacements.

Cost is a common criterion in civil engineering. The tensegrity bridge is a steel structure. The cost in steel construction is defined mostly by the joints. The pentagon module has the lowest number of joints compared with other continuous ring modules. Therefore, it is not only the best performing module but it is likely to be the most economical configuration.

\section{Conclusions}

Tensegrity-structure design is a challenging task due to geometrical complexity and closely coupled behavior. For applications of tensegrity ring modules in large-scale construction such as bridges, this work results in the following conclusions.

- A novel tensegrity bridge has been developed through a parametric design study of three potential module configurations: square, pentagon and hexagon. All modules were designed under similar criteria related to span, internal pedestrian space and loading. A structural efficiency index takes into account self-weight and deflection to assess the ability of these modules to satisfy design criteria. It was shown that the most efficient module is the pentagon ring and the least efficient is the hexagon.

- The existence of helical strut topologies in the ring configuration and the observation of differential vertical displacements confirm that there is torsion within the structure. The cause of torsion is the lack of symmetry about the $y-z$ plane (x: longitudinal axis and $z$ : vertical axis). However, feasible configurations with negligible effects of torsion can be found.

- A useful extension of the tensegrity grid design guideline includes the ratio of rigidity between cables and struts as well as material properties. Increasing this ratio decreases vertical displacements until reaching a limit that varies with material properties.

Tensegrity structures are efficient when properly designed. Further work involves construction and testing a prototype of the pentagon tensegrity bridge.

\section{Acknowledgments}

Authors would like to thank the Swiss National Science Foundation for supporting this work through contract number 200020-121552/1. They are also grateful to Dr. Prakash Kripakaran for discussions.

\section{Appendix}

A. Topology of the modules

Table 9 presents the nodal coordinates for a single module of the configurations studied based on the construction method of Section 3. 
Table 9: Nodal coordinates of single ring modules

\begin{tabular}{|c|c|c|c|c|c|c|c|c|c|}
\hline \multirow[b]{2}{*}{ Node } & \multicolumn{3}{|c|}{ Square } & \multicolumn{3}{|c|}{ Pentagon } & \multicolumn{3}{|c|}{ Hexagon } \\
\hline & $\mathrm{x}[\mathrm{cm}]$ & $\mathrm{y}[\mathrm{cm}]$ & $\mathrm{z}[\mathrm{cm}]$ & $\mathrm{x}[\mathrm{cm}]$ & $\mathrm{y}[\mathrm{cm}]$ & $\mathrm{z}[\mathrm{cm}]$ & $\mathrm{x}[\mathrm{cm}]$ & $\mathrm{y}[\mathrm{cm}]$ & $\mathrm{z}[\mathrm{cm}]$ \\
\hline 1 & 0.00 & 0.00 & 0.00 & 0.00 & 0.00 & 389.40 & 0.00 & -327.42 & -67.04 \\
\hline 2 & 0.00 & 520.00 & 0.00 & 0.00 & 370.30 & 120.30 & 0.00 & -128.72 & -67.04 \\
\hline 3 & 0.00 & 520.00 & 520.00 & 0.00 & 228.90 & -315.00 & 0.00 & -29.37 & 105.03 \\
\hline 4 & 0.00 & 0.00 & 520.00 & 0.00 & -228.90 & -315.00 & 0.00 & -128.72 & 277.11 \\
\hline 5 & 250.00 & 260.00 & -107.70 & 0.00 & -370.30 & 120.30 & 0.00 & -327.42 & 277.11 \\
\hline 6 & 250.00 & 627.70 & 260.00 & 250.00 & 0.00 & -389.40 & 0.00 & -426.77 & 105.03 \\
\hline 7 & 250.00 & 260.00 & 627.70 & 250.00 & -370.30 & -120.30 & 250.00 & -228.07 & -93.66 \\
\hline 8 & 250.00 & -107.70 & 260.00 & 250.00 & -228.90 & 315.00 & 250.00 & -55.99 & 5.68 \\
\hline 9 & 500.00 & 0.00 & 0.00 & 250.00 & 228.90 & 315.00 & 250.00 & -400.15 & 5.68 \\
\hline 10 & 500.00 & 520.00 & 0.00 & 250.00 & 370.30 & -120.30 & 250.00 & -55.99 & 204.38 \\
\hline 11 & 500.00 & 520.00 & 520.00 & 500.00 & 0.00 & 389.40 & 250.00 & -400.15 & 204.38 \\
\hline 12 & 500.00 & 0.00 & 520.00 & 500.00 & 370.30 & 120.30 & 250.00 & -228.07 & 303.73 \\
\hline 13 & - & - & - & 500.00 & 228.90 & -315.00 & 500.00 & -327.42 & -67.04 \\
\hline 14 & - & - & - & 500.00 & -228.90 & -315.00 & 500.00 & -128.72 & -67.04 \\
\hline 15 & - & - & - & 500.00 & -370.30 & 120.30 & 500.00 & -29.37 & 105.03 \\
\hline 16 & - & - & - & - & - & - & 500.00 & -128.72 & 277.11 \\
\hline 17 & - & - & - & - & - & - & 500.00 & -327.42 & 277.11 \\
\hline 18 & - & - & - & - & - & - & 500.00 & -426.77 & 105.03 \\
\hline
\end{tabular}

Table 10: Connectivity of single ring modules

\begin{tabular}{lcccccc}
\hline & & \multicolumn{2}{c}{ Square } & \multicolumn{2}{c}{ Pentagon } & \multicolumn{2}{c}{ Hexagon } \\
Element & Node & Node & Node & Node & Node & Node \\
\hline 1 & 1 & 2 & 1 & 2 & 1 & 2
\end{tabular}




\begin{tabular}{|c|c|c|c|c|c|}
\hline 2 & 2 & 3 & 2 & 3 & 2 \\
\hline 3 & 3 & 4 & 3 & 4 & 3 \\
\hline 4 & 4 & 1 & 4 & 5 & 4 \\
\hline 5 & 9 & 5 & 5 & 1 & 5 \\
\hline 6 & 10 & 5 & 11 & 12 & 6 \\
\hline 7 & 2 & 5 & 12 & 13 & 7 \\
\hline 8 & 1 & 5 & 13 & 14 & 7 \\
\hline 9 & 2 & 6 & 14 & 15 & 8 \\
\hline 10 & 10 & 6 & 15 & 11 & 8 \\
\hline 11 & 11 & 6 & 1 & 9 & 9 \\
\hline 12 & 3 & 6 & 11 & 9 & 9 \\
\hline 13 & 4 & 7 & 2 & 9 & 10 \\
\hline 14 & 3 & 7 & 12 & 9 & 10 \\
\hline 15 & 11 & 7 & 2 & 10 & 11 \\
\hline 16 & 12 & 7 & 12 & 10 & 11 \\
\hline 17 & 9 & 8 & 3 & 10 & 12 \\
\hline 18 & 1 & 8 & 13 & 10 & 12 \\
\hline 19 & 4 & 8 & 3 & 6 & 14 \\
\hline 20 & 12 & 8 & 13 & 6 & 13 \\
\hline 21 & 9 & 10 & 4 & 6 & 14 \\
\hline 22 & 10 & 11 & 14 & 6 & 15 \\
\hline 23 & 11 & 12 & 4 & 7 & 13 \\
\hline 24 & 12 & 9 & 14 & 7 & 18 \\
\hline 25 & 1 & 6 & 5 & 7 & 15 \\
\hline 26 & 6 & 12 & 15 & 7 & 16 \\
\hline 27 & 12 & 1 & 5 & 8 & 18 \\
\hline 28 & 2 & 7 & 15 & 8 & 17 \\
\hline 29 & 7 & 9 & 1 & 8 & 16 \\
\hline 30 & 9 & 2 & 11 & 8 & 17 \\
\hline
\end{tabular}




\begin{tabular}{|c|c|c|c|c|c|c|}
\hline 31 & 3 & 8 & 1 & 12 & 13 & 14 \\
\hline 32 & 8 & 10 & 2 & 13 & 14 & 15 \\
\hline 33 & 10 & 3 & 3 & 14 & 15 & 16 \\
\hline 34 & 4 & 5 & 4 & 15 & 16 & 17 \\
\hline 35 & 5 & 11 & 5 & 11 & 17 & 18 \\
\hline 36 & 11 & 4 & 15 & 9 & 18 & 13 \\
\hline 37 & - & - & 9 & 3 & 6 & 7 \\
\hline 38 & - & - & 11 & 10 & 4 & 11 \\
\hline 39 & - & - & 10 & 4 & 5 & 9 \\
\hline 40 & - & - & 12 & 6 & 1 & 8 \\
\hline 41 & - & - & 6 & 5 & 2 & 10 \\
\hline 42 & - & - & 13 & 7 & 3 & 12 \\
\hline 43 & - & - & 7 & 1 & 16 & 8 \\
\hline 44 & - & - & 14 & 8 & 15 & 7 \\
\hline 45 & - & - & 8 & 2 & 14 & 9 \\
\hline 46 & - & - & - & - & 13 & 11 \\
\hline 47 & - & - & - & - & 18 & 12 \\
\hline 48 & - & - & - & - & 17 & 10 \\
\hline 49 & - & - & - & - & 13 & 2 \\
\hline 50 & - & - & - & - & 14 & 3 \\
\hline 51 & - & - & - & - & 15 & 4 \\
\hline 52 & - & - & - & - & 16 & 5 \\
\hline 53 & - & - & - & - & 18 & 1 \\
\hline 54 & - & - & - & - & 17 & 6 \\
\hline
\end{tabular}

B. Load combinations and limit-state checks

Table 11 contains the load combinations for the tensegrity footbridge according to Swiss SIA codes for the ultimate limit state and the service limit state. Table 12 and 13 contain the ultimate limit-state 
checks and the serviceability limit-state requirements resepectively for the tensegrity footbridge according to Swiss SIA.

Table 11: Loads factors of the critical load model

\begin{tabular}{lllll}
\hline \multicolumn{2}{l}{ Ultimate limit state } & \multicolumn{2}{l}{ Service limit state } \\
Load & Safety factor & Base value & Safety factor & Base value \\
\hline Dead load & 1.35 & $78.5 \mathrm{kN} / \mathrm{m}^{3}$ & 1.0 & $78.5 \mathrm{kN} / \mathrm{m}^{3}$ \\
$\begin{array}{l}\text { Vertical service load } \\
\text { Horizontal service }\end{array}$ & 1.5 & $4 \mathrm{kN} / \mathrm{m}^{2}$ & 1.0 & $4 \mathrm{kN} / \mathrm{m}^{2}$ \\
load & 1.5 & $\begin{array}{l}10 \% \text { of the vertical } \\
\text { service load }\end{array}$ & 1.0 & $\begin{array}{l}10 \% \text { of the vertical } \\
\text { service load }\end{array}$ \\
\hline
\end{tabular}

The wind load is not considered as this study is limited to static design criteria.

Table 12: Ultimate limit-state checks

\begin{tabular}{ll}
\hline & Ultimate limit state: $\mathrm{E}_{\mathrm{d}} \leq \mathrm{R}_{\mathrm{d}}$ \\
\hline Tension components & Compression components \\
Tensile resistance & Buckling resistance \\
$E_{d} \leq \frac{f_{y} A}{1.05}$ & $E_{d} \leq \chi_{k} \frac{f_{y} A}{1.05}$ \\
\hline
\end{tabular}

where $E_{d}$ is the design value of an action effect, $R_{d}$ is the design value of the ultimate resistance, $f_{y}$ the yield strength, $A$ the cross-sectional area of the element and $\chi_{k}$ the reduction factor for buckling. For serviceability reasons compression structural components is also verified as follows:

$$
\frac{l_{k}}{r_{\min }} \leq 200
$$

where $l_{k}$ is the effective length of strut and $r_{\min }$ the minimum radius of gyration.

Table 13: Serviceability limit-state requirements (SIA-260)

Criterion Limit $\quad$ Value for a $20 \mathrm{~m}$ span


Rhode-Barbarigos, L., Bel Hadj Ali, N., Motro, R. and Smith, I.F.C. "Designing tensegrity modules for pedestrian bridges" Engineering Structures, Vol.32, No.4, 2010, pp 1158-1167.

\begin{tabular}{lll}
\hline Functionality & $w \leq L / 700$ & $2.85 \mathrm{~cm}$ \\
Comfort & $w \leq L / 600$ & $3.33 \mathrm{~cm}$ \\
Appearance & $w \leq L / 700$ & $2.85 \mathrm{~cm}$ \\
\hline
\end{tabular}

where $L$ is the footbridge span. 


\section{References}

[1] Motro R. Tensegrity: Structural systems for the future. Hermes ed. U.K. 2003.

[2] Hanaor A. Geometrically Rigid Double-Layer Tensegrity Grids. International Journal of Space Structures. 1994;9:227-38.

[3] Skelton RE, Adhikari R, Pinaud JP, Waileung C, Helton JW. An introduction to the mechanics of tensegrity structures. Proceedings of the 40th IEEE Conference on Decision and Control. Orlando, FL, USA 2001:4254-9 vol.5.

[4] Tibert AG. Deployable tensegrity structures for space applications. Stockholm: Royal Institute of Technology 2002.

[5] Paronesso A, Passera R. The cloud of Yverdon. IASS Symposium 2004. 2004:184-5.

[6] Paronesso A. The 2002 World Cycling Center Arena Aigle, Switzerland. International IASS

Symposium on "Lightweight Structures in Civil Engineering". Warsaw, Poland 2002.

[7] Klimke H, Stephan S. The making of a tensegrity tower. IASS 2004 Symposium, International Association for Shell and Spatial Structures. Montpellier: Editions de l'Espérou 2004.

[8] Schlaich M. The messeturm in Rostock - A tensegrity tower. Journal of the International Association for Shell and Spatial Structures. 2004;45(145):93-8.

[9] Wang BB. Free-Standing Tension Structures From Tensegrity Systems to Cable-Strut Systems Routledge, UK 2004.

[10] Motro R. Tensegrity systems: the state of the art. International Journal of Space Structures. 1992;7(2):75-82.

[11] Hanaor A. Aspects of Design of Double-Layer Tensegrity Domes. International Journal of Space Structures. 1992;7:101-13.

[12] Quirant J, Kazi-Aoual MN, Motro R. Designing tensegrity systems: the case of a double layer grid. Engineering Structures. 2003;25(9):1121-30.

[13] Tibert G. Deployable tensgrity structures for space applications. PhD Thesis, Royal Institute of Technology, Stckholm, Sweden 2002.

[14] Tibert AG, Pellegrino S. Deployable tensegrity mast. 44th AIAA/ASME/ASCE/AHS/ASC, Structures, Structural Dynamics and Materials Conference and Exhibit. Norfolk, VA, USA 2003.

[15] Fest E, Shea K, Smith IFC. Active Tensegrity Structure. Journal of Structural Engineering. 2004;130(10):1454-65.

[16] Domer B, Fest E, Lalit V, Smith IFC. Combining Dynamic Relaxation Method with Artificial Neural Networks to Enhance Simulation of Tensegrity Structures. Journal of Structural Engineering. 2003;129(5):672-81.

[17] Domer B, Smith IFC. An Active Structure that Learns. Journal of Computing in Civil Engineering. 2005;19(1):16-24.

[18] Adam B, Smith IFC. Self-Diagnosis and Self-Repair of an Active Tensegrity Structure. Journal of Structural Engineering. 2007;133(12):1752-61.

[19] Adam B, Smith IFC. Reinforcement Learning for Structural Control. Journal of Computing in Civil Engineering. 2008;22(2):133-9.

[20] Smaili A, Motro R. Foldable/unfordable curved tensegrity systems by finite mechanism activation. Journal of the International Association for Shell and Spatial Structures. 2007;48(155):15360 .

[21] Vu KK, Richard Liew JY, Anandasivam K. Deployable tension-strut structures: from concept to implementation. Journal of Constructional Steel Research. 2006;62(3):195-209.

[22] Motro R, Maurin B, Silvestri C. Tensegrity Rings and the Hollow Rope. IASS Symposium 2006, New Olympics, New Shells and Spatial Structures. Beijing 2006:470-1.

[23] Pugh A. An Introduction to Tensegrity: University of California Press 1976.

[24] Pellegrino S, Calladine CR. Matrix analysis of statically and kinematically indeterminate frameworks. International Journal of Solids and Structures. 1986;22(4):409-28.

[25] Nishimura Y, Murakami H. Initial shape-finding and modal analyses of cyclic frustum tensegrity modules. Computer Methods in Applied Mechanics and Engineering. 2001;190(4344):5795-818. 
[26] Pellegrino S. Mechanics of kinematically indeterminate structures. PhD Thesis, University of Cambridge, U.K., 1986.

[27] Barnes MR. Form Finding and Analysis of Tension Structures by Dynamic Relaxation. International Journal of Space Structures. 1999;14:89-104.

[28] Vassart N, Motro R. Multiparametered Formfinding Method: Application to Tensegrity Systems. International Journal of Space Structures. 1999;14:147-54.

[29] Paul C, Lipson H, Cuevas FV. Evolutionary form-finding of tensegrity structures. Genetic and Evolutionary Computation Conference. Washington D.C. 2005:3-10.

[30] Tibert AG, Pellegrino S. Review of Form-Finding Methods for Tensegrity Structures. International Journal of Space Structures. 2003;18:209-23.

[31] Juan SH, Mirats Tur JM. Tensegrity frameworks: Static analysis review. Mechanism and Machine Theory. 2008;43(7):859-81.

[32] Kebiche K, Kazi-Aoual MN, Motro R. Geometrical non-linear analysis of tensegrity systems. Engineering Structures. 1999;21(9):864-76.

[33] Day AS. An introduction to dynamic relaxation. The Engineer. 1965;219:218-21.

This work is licensed under a Creative Commons Attribution-NonCommercial-NoDerivatives 4.0 International License

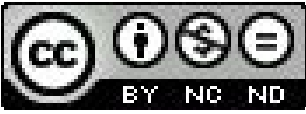

